\title{
Highly stretchable metal-polymer hybrid conductors for wearable and self-cleaning sensors
}

\author{
Sunyoung Yoon', Yong Jun Kim', Yu Rim Lee', Nae-Eung Lee®i', Yoochan Won', Srinivas Gandla', \\ Sunkook Kim (1) ${ }^{1}$ and Han-Ki Kim (1)
}

\begin{abstract}
We fabricated semitransparent and stretchable hybrid Ag-polytetrafluoroethylene (PTFE) conductors on a polyurethane (PU) substrate for use in high-performance wearable and self-cleaning sensors. The highly conductive Ag metal and stretchable PTFE polymer matrix were cosputtered, embedding the self-formed Ag in the PTFE matrix. Depending on the cosputtering RF and DC power ratio, the Ag-PTFE conductors showed a sheet resistance of 3.09-17.23 $\Omega /$ square and an optical transparency of $25.27-38.49 \%$ at a wavelength of $550 \mathrm{~nm}$. Under the optimal cosputtering conditions, the Ag-PTFE electrode showed outstanding stretchability (strain 20\%) and reversible hysteresis, enabling the production of stretchable and semitransparent conductors. In addition, the very small critical inward/outward bending radius near $1 \mathrm{~mm}$ and the hydrophobic surface indicate that the Ag-PTFE films could also be applied in wearable and self-cleaning devices. The suitability of the high stretchability and low sheet resistance of the sputtered Ag-PTFE conductor was verified by using it as a stretchable interconnector for commercial ELs, LEDs, and strain sensors. We applied the Ag-PTFE film as a semitransparent conductor for stretchable touch panels and electromyography sensors. Cosputtered Ag-PTFE films are promising stretchable conductors for a variety of applications in next-generation wearable devices.
\end{abstract}

\section{Introduction}

Wearable and stretchable electronics for human or robot bodies are becoming increasingly popular for their potential uses in healthcare monitoring, artificial robots, eye cameras, and electronic skin ${ }^{1-6}$. The weakest parts in wearable and stretchable electronics are the stretchable conductors because typical metal or inorganic electrodes are easily cracked by external stretching ${ }^{7,8}$. Therefore, wearable electronics require highly conductive, stretchable electrode materials, which can be prepared via simple, scalable physical vapor deposition as part of a typical device fabrication process. Until now, most wearable and stretchable electronics for skin or the human body have been fabricated on elastomeric substrates such as polyurethane (PU), styrene-butadiene

Correspondence: Han-Ki Kim (hankikim@skku.edu)

${ }^{1}$ School of Advanced Materials Science and Engineering, Sungkyunkwan University, 2066, Seobu-ro, Jangan-gu, Suwon-si, Gyeonggi-do 16419,

Republic of Korea rubber (SBR), Ecoflex, and polydimethylsiloxane (PDMS), which have high stretchability ${ }^{9-12}$. Compared to the stretchability of the substrate, metal or transparent conducting oxide (TCO) electrodes showed poor stretchability resulting from the small strain failure values of the metal or TCO materials. To overcome the low stretchability of of metal or TCO electrodes, several hybrid electrodes consisting of two kinds of electrode materials, such as graphene, carbon nanotubes (CNTs), reduced graphene oxide nanosheets, conductive polymers, nanoparticles, and nanowires, have been reported as stretchable electrodes ${ }^{13-19}$. Zhow et al. ${ }^{20}$ reported on a stretchable CNT-polyacrylic acid hybrid electrode with a sheet resistance of $60 \Omega$ /square for use as electrodes in wearable electronic devices and bioelectronics and stretching of $40 \%$ strain. Fan et al. ${ }^{21}$ also reported on a high-performance Ag NW-PEDOT:PSS hybrid electrode embedded in an elastomer with a gauge factor (GF) of 6.5-8.0. Although there have been many reports on

\section{(c) The Author(s) 2021}

(c) Open Access This article is licensed under a Creative Commons Attribution 4.0 International License, which permits use, sharing, adaptation, distribution and reproduction in any medium or format, as long as you give appropriate credit to the original author(s) and the source, provide a link to the Creative Commons license, and indicate if changes were made. The images or other third party material in this article are included in the article's Creative Commons license, unless indicated otherwise in a credit line to the material. If material is not included in the article's Creative Commons license and your intended use is not permitted by statutory regulation or exceeds the permitted use, you will need to obtain permission directly from the copyright holder. To view a copy of this license, visit http://creativecommons.org/licenses/by/4.0/. 
hybrid stretchable electrodes that can enhance the performance of wearable electronics, most of those previous reports have been achieved using complicated, expensive, small-area procedures, including a complicated transfer solution and additional processing (plasmonic welding, multistep processes) $)^{22-25}$. For the mass production of wearable electronics, a large-area coating process is needed to fabricate stretchable electrodes compatible with current thin-film processes, such as sputtering or evaporation. Although solution-based printing technologies have been applied in large-area coating processes, vacuum-based sputtering or roll-to-roll sputtering is still suitable for preparing large-area flexible electrodes due to its uniformity and reproducibility. Since most components in stretchable electronics are compatible with current semiconductor-based devices, the fabrication process is also compatible with a current semiconductor fabrication process. In particular, the large-area coating and fabrication of wearable and stretchable electronics should employ typical magnetron sputtering-based coatings. Although some stretchable electrodes with typical 2- or 3-dimensional structures have been fabricated by conventional sputtering or evaporation, a detailed investigation of stretchable electrodes fabricated by the cosputtering process for stretchable sensors or electrodes is still needed.

In this work, we demonstrate semitransparent and highly stretchable Ag-PTFE composite conductors for stretchable interconnectors, touch panels, and sensors. High-performance stretchable conductors are fabricated by employing typical direct current (DC) and radio frequency (RF) magnetron cosputtering, which is appropriate for current nano and microelectronic fabrication. In the hybrid structure, the Ag acts as a conductive percolation path, and the PTFE matrix serves to control the agglomeration of $\mathrm{Ag}$ and to improve the mechanical stretchability of the hybrid conductors. The optical, electrical, morphological, and mechanical properties of the PTFE-modified Ag hybrid conductor were investigated as a function of the ratio of RF and DC power simultaneously applied to the polymer PTFE and metal Ag targets. To show the feasibility of using the Ag-PTFE conductor as interconnectors, commercial electroluminescence (EL) and light-emitting diodes (LEDs) were connected to the Ag-PTFE conductors, showing operation under stretched conditions. In addition, the stretchable Ag-PTFE conductors were employed in strain sensors, capacitive touch panels, and electromyography (EMG) sensors. The successful operation of the strain sensor, touch panels, and EMG under stretching conditions indicated that the Ag-PTFE hybrid conductor fabricated by cosputtering is a promising stretchable conductor that can replace current hybrid stretchable conductors fabricated by expensive and complicated fabrication processes that are not suitable for large-area device fabrication.

\section{Experimental procedures Preparation of PTFE-modified Ag hybrid conductors on a PU substrate}

The PTFE-modified Ag hybrid conductor was sputtered onto a $140 \mu \mathrm{m}$ PU substrate using a magnetron sputtering system at a low temperature $\left(30-45^{\circ} \mathrm{C}\right.$, Fig. S1, Supplementary Information). The PTFE:CNT (JAEWOO) and Ag (99.99\%, Dasom RMS) targets were attached to tiled cathode guns and cosputtered using an RF and DC power source. PTFE powder (A7-J, DuPont Mitsui) and multiwall CNT powder (HANOS CM-280, Hanwha Chemical) were premixed with PTFE: CNT weight ratios of 95:5. The $\mathrm{Ag}$ and PTFE films were cosputtered with a base pressure of $<3 \times 10^{-6}$ Torr, working pressure of $4 \mathrm{mTorr}$, and an Ar flow rate of $20 \mathrm{sccm}$. The deposition thickness was $20 \mathrm{~nm}$, which was confirmed with a surface profiler (NanoMap LS, AEP Technology).

\section{Analysis of the Ag-PTFE hybrid conductors}

The optical and electrical properties of the Ag-PTFE conductor were investigated using a UV-visible spectrometer (V-670, Jasco) and a Hall measurement (HMS4500AM, Ecopia). The electrical resistivity of the optimized Ag-PTFE conductor was measured using a physical property measurement system (Quantum Design) in the temperature range from 2 to $300 \mathrm{~K}$ to confirm the conduction mechanism. A contact angle analyzer (PhoenixMT (A), SEO CO.) was used to calculate the surface energy of the Ag-PTFE film. Three milliliters of deionized water and diiodomethane served as the polar and dispersed components, and the surface energy was calculated. The surface morphology and microstructure of the Ag-PTFE conductors were investigated using field emission scanning electron microscopy (FESEM: JSM-7600F, JEOL) and transmission electron microscopy (TEM: JEM2100F, JEOL). The mechanical properties of the Ag-PTFE films were analyzed using our lab-designed stretching and inner/outer bending test system. To confirm the mechanical stability, we performed the dynamic fatigue stretching test at $20 \%$ strain and the dynamic fatigue bending test at a bending radius of $1 \mathrm{~mm}$ for 1000 cycles.

\section{Fabrication of the stretchable strain sensor and EL/LED interconnector}

Optimized stretchable Ag-PTFE conductors were fabricated with DC:RF $(5: 40 \mathrm{~W})$ conditions on a PU substrate with an area of $1.5 \times 5 \mathrm{~cm}^{2}$. The operation of the strain sensor attached to the wrist of the human body was monitored by using a digital multimeter (DMM: $34470 \mathrm{~A}$, Keysight). When stress was applied to the strain sensor, the resistance of the Ag-PTFE conductors changed due to 
the elongation of the Ag-PTFE conductors. These changes in resistance could detect the deformation applied to the sensor. In addition, to verify the stability, repeated strain sensing was performed 100 times between $10 \%$ strain and $20 \%$ strain by connecting the DMM and a lab-designed stretching test system. To confirm the application as stretchable interconnectors, the Ag-PTFE conductor was connected to an electroluminescent (EL: National EL technology) and a LED.

\section{Fabrication of a stretchable touch sensor}

The size of the touch screen sensor was set to $5 \times 3 \mathrm{~cm}^{2}$. We used a metal mask to deposit the patterned Ag-PTFE film as a bottom electrode on the PU substrate for the touch sensor. After depositing the bottom electrode on the PU substrate, the contact area was covered with PI tape and spin-coated with PU. The dielectric layer of the touch sensor was formed by spin coating PU (Alberdingk U3251, Alberdingk Boley) dispersed in water. The PU dispersion was spin-coated on the bottom electrode at $2000 \mathrm{rpm}$ for $60 \mathrm{~s}$ and dried at $60^{\circ} \mathrm{C}$ for $4 \mathrm{~h}$. The top electrode on the PU was coated at $2000 \mathrm{rpm}$ for $15 \mathrm{~s}$ using PDMS as encapsulation. To minimize contact damage to the touch sensor when sensing capacitance, Au textiles were used as electrodes. The capacitance measurement to detect touch used an AC pulse (with an amplitude of -5 to $5 \mathrm{~V}$ and a frequency of $10 \mathrm{~Hz}$ ) to the top Ag-PTFE conductor to induce a dipole alignment inside the $\mathrm{PU}$ dielectric layer using a Semiconductor Device Analyzer (B1500A, Keysight).

\section{Measurement of the EMG sensor}

Solution-based polyimide (PI) (CAS \# 872-50-4) was purchased from HD-MicroSystems and used as received. First, solution-based PI was spin-coated on a $4 \times 4 \mathrm{~cm}^{2}$ precleaned silicon substrate at $2000 \mathrm{rpm}$ for $30 \mathrm{~s}$ as a supporting layer for the sensor. After that, the substrates were soft baked at $90^{\circ} \mathrm{C}$ for $5 \mathrm{~min}$ and then cured at $350^{\circ} \mathrm{C}$ for $1 \mathrm{~h}$ on a hot plate. The Ag-PTFE conductor was deposited on the substrates with DC $5 \mathrm{~W}: \mathrm{RF} 40 \mathrm{~W}$ conditions by cosputtering. Next, the Ag-PTFE layer and PI substrate were completely ablated along with the thickness by using a programmable bench-top infrared (IR) laser machine to obtain the desired pattern. Then, the patterned sensor was self-detached from the silicon substrate just by dipping in hot water maintained at $90^{\circ} \mathrm{C}$, and it was later transferred onto the glass substrate while floating on water. Thereafter, anisotropic conductive film bonding was conducted between the patterned sensor and the flexible conductive wire, helping the sensor to draw signals without any noise during EMG monitoring. Finally, a sticky and stretchable PDMS (polydimethylsiloxane)/PEIE (polyethyleneimine $80 \%$ ethoxylated solution) patch (1$\mathrm{mm}$ thick) was attached on top of the sensor. Herein, the
PDMS-PEIE patch was prepared by mixing $10 \mathrm{~g}$ of PDMS base agent to $1 \mathrm{~g}$ of PDMS curing agent with $40 \mu \mathrm{l}$ of PEIE added to it, and then the mixture was degassed and cured at $90^{\circ} \mathrm{C}$ for $4 \mathrm{~h}$. The EMG measurements were performed by using an MP36 (Biopac System, Inc., USA).

\section{Results and discussion}

The semitransparent and highly stretchable Ag-PTFE conductors were prepared by the magnetron cosputtering of 3-inch Ag metal and PTFE polymer targets on a 140$\mu \mathrm{m}$-thick commercial PU substrate at room temperature (Fig. 1a). In particular, to sputter the insulating PTFE target, we employed a specially designed PTFE:CNT mixed target (PTFE: CNT weight ratio 95:5) ${ }^{26}$. Most stretchable conductors are currently fabricated on thick PDMS substrates, but such a PDMS substrate requires a complicated preparation procedure ${ }^{27}$. We were able to fabricate our conductors easily and quickly using commercially available transparent PU substrates. During cosputtering of the metal (Ag) and PTFE (polymer) targets, sputtered $\mathrm{Ag}$ atoms and $\mathrm{C}-\mathrm{F}$ chains were randomly deposited on the PU substrate and formed a randomly connected Ag path in the PTFE matrix, as shown in Fig. 1b. Because of the low Young's modulus of the PTFE matrix and good stretchability of the Ag network structure, the PTFE-Ag hybrid was stretchable. Those randomly connected Ag networks embedded in the PTFE matrix maintained their conducting path, even under stretching, due to the high stretchability of the PTFE matrix. Since the Ag-PTFE conductor shows unique electrical and mechanical properties depending on the ratio of DC to RF power applied on the Ag and PTFE targets, we optimized the Ag-PTFE conductor based on the DC/RF power ratio. The optimized Ag-PTFE conductors could be applied in wearable and stretchable sensors, such as wearable strain sensors, wearable touch screen panels, and wearable EMG sensors, as shown in Fig. 1c. To apply Ag-PTFE conductors in wearable and self-cleaning sensors, an incomplete wetting surface is important for the hybrid electrode. Therefore, the contact angle and surface energy of the Ag-PTFE conductors were measured as a function of the DC and RF power applied to Ag and PTFE targets.

Figure $2 \mathrm{a}$ shows a picture of the contact angle as a function of the DC and RF power ratio. An increase in the RF power applied to the PTFE target leads to an increase in the contact angle of the hybrid electrodes due to the increase in the PTFE portion with hydrophobic properties. The droplet shape on the Ag-PTFE conductor indicates the formation of an incomplete wetting surface on the hybrid electrodes. Figure $2 b$ shows the surface energy of the Ag-PTFE conductor as a function of the DC/RF power ratio. The surface energy was calculated using the contact angle measurement (Phoenix-MT(A), SEO CO.) 


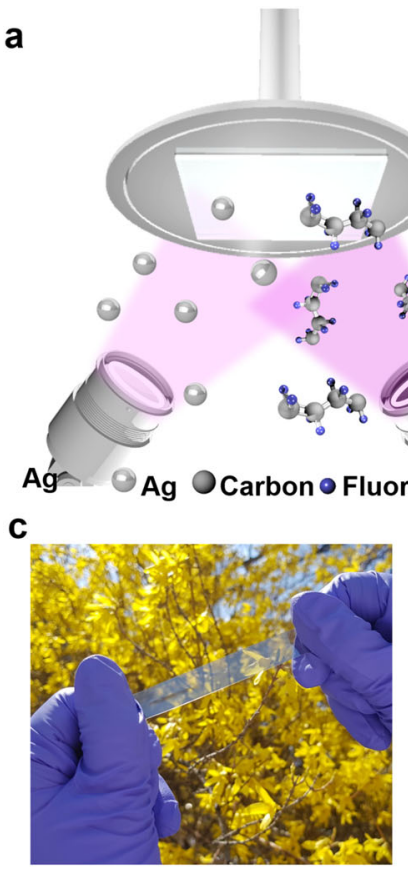

Stain sensor

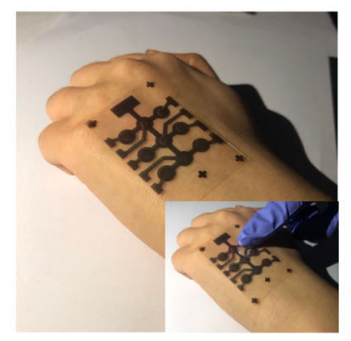

Wearable touch sensor

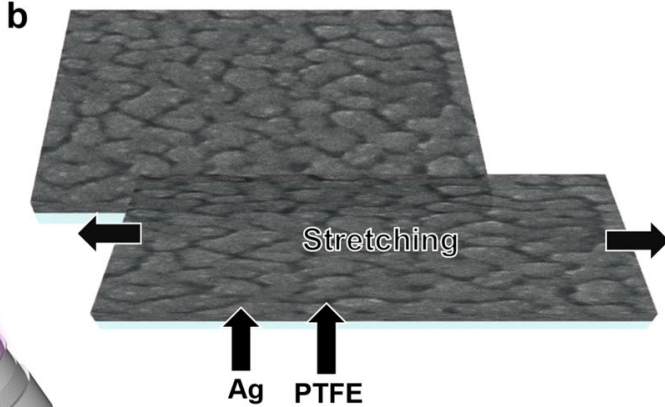

PRFE

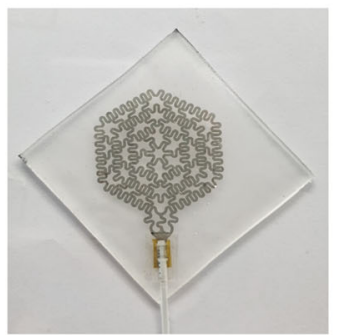

EMG sensor

Fig. 1 Fabrication and applications of Ag-PTFE hybrid conductors. a Schematics of Ag and PTFE cosputtering to fabricate stretchable hybrid electrodes. b Surface FESEM image of the Ag-PTFE hybrid conductor before and after stretching. c Promising applications of the stretchable and semitransparent Ag-PTFE hybrid conductor in strain sensors, wearable touch sensors, and EMG sensors.

as a function of the $\mathrm{DC} / \mathrm{RF}$ power ratio. The contact angle of the surface could be expressed using Young's equation as follows ${ }^{28}$.

$$
\cos \theta=\left(\gamma_{F}-\gamma_{\mathrm{FL}}\right) / \gamma_{L}
$$

where $\gamma_{F}$ is the interfacial free energy of the film-vapor interface, $\gamma_{\mathrm{FL}}$ is the interfacial free energy of the filmliquid interface, and $\gamma_{L}$ is the interfacial free energy of the liquid-vapor interface. The surface energy was calculated using the Owens, Wendt, Rabel, and Kaelble (OWRK) methods. The contact angle was measured at five or more random points, and the average value is indicated. By measuring the contact angle using two solutions with known surface energy components, it is possible to separate the dispersion component of the surface energy from the polar component. In this work, a polar solution (deionized water) and a dispersion solution (diiodomethane) were used for the calculations. According to the Fowkes approximation below, the surface energy of each component was calculated ${ }^{29}$.

$$
\gamma_{F}=\gamma_{F}^{d}+\gamma_{F}^{p}
$$

In the above equation, $d$ and $p$ represent the dispersive force and hydrogen bonding components, respectively.
The surface energy $\left(\gamma_{F}\right)$ of the Ag-PTFE conductor can explain the sum that was calculated using Eq. (2). As shown in Fig. 2b, an increase in the RF power of PTFE resulted in a significant decrease in the surface energy, while the DC power increase led to a small decrease in the surface energy. Table 1 shows the surface energy of the Ag-PTFE conductor as a function of the DC/RF power ratio. This is because the strong interactions between $C$ and F of PTFE form an incomplete wetting surface on the manufactured sample, as shown in Fig. 2c. Although the Ag-PTFE hybrid electrode showed a smaller contact angle $\left(64.53^{\circ}\right)$ than that $\left(106.69^{\circ}\right)$ of the bare PTFE film (Fig. S2a), the high contact angle of the Ag-PTFE hybrid electrode is enough to use the product as a self-cleaning electrode for wearable electronics. The water drops on the surface of the Ag-PTFE hybrid electrode could maintain a clean surface since the water drops were repelled from the surface of the Ag-PTFE-based wearable device, as shown in Fig. S2b and video (Supporting information) showing the self-cleaning surface of the Ag-PTFE hybrid electrode. The surface morphology and agglomeration of Ag in the hybrid conductor were critically dependent on the DC/RF power ratio, as shown in Fig. 2d. As expected from the contact angle, the increase in the RF power for PTFE led to a self-agglomerated Ag network due to the hydrophobic surface of the PTFE matrix. Compared to the AgPTFE conductor prepared at a DC/RF ratio of $3 / 10 \mathrm{~W}$, the 


\section{a}

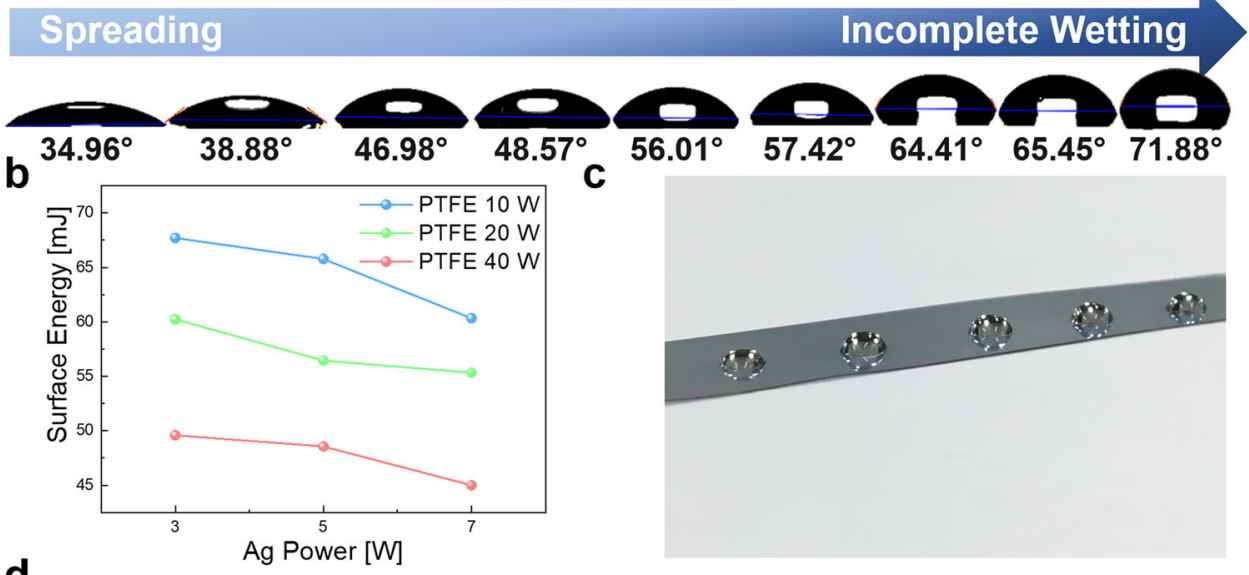

d
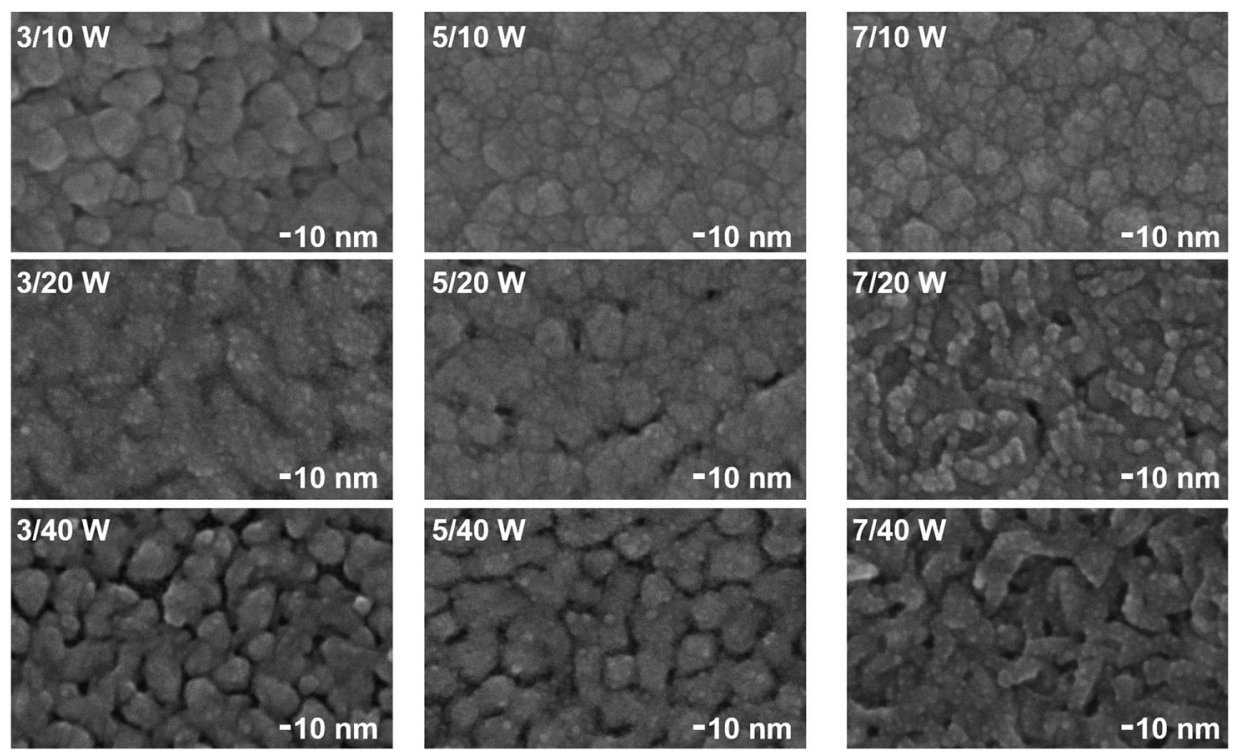

Fig. 2 Images of the Ag-PTFE conductor and surface energy as a function of DC and RF power applied in the Ag and PTFE targets. a Photograph showing the contact angle as a function of the DC/RF power ratio. $\mathbf{b}$ Surface energy of the Ag-PTFE conductor as a function of the DC/ RF power ratio. c The photograph illustrates a water droplet on the Ag-PTFE hybrid conductor prepared at a DC/RF power of 5/40 W. $\mathbf{d}$ FESEM images of Ag-PTFE hybrid conductors as a function of DC/RF power ratio.

Ag-PTFE conductor prepared at a DC/RF power of 3/ $40 \mathrm{~W}$ showed a more agglomerated Ag network, which is beneficial for stretching. Table 2 shows the sheet resistance and conductivity of the Ag-PTFE conductor according to the DC/RF power ratios as well as the $20 \mathrm{~nm}$ thick pure Ag film. As summarized in Table 2, the sputtered pure Ag film showed much higher conductivity than the Ag-PTFE hybrid films. The sheet resistance of the 20$\mathrm{nm}$ thick pure $\mathrm{Ag}$ film is $2.53 \Omega$ /square, and the conductivity is $197,460 \mathrm{~S} / \mathrm{cm}$ (inverse of resistivity). In contrast to the contact angle, the sheet resistance of the AgPTFE conductors is significantly dependent on the DC power for Ag sputtering. With an increase in the DC power at the same RF power of the PTFE, the Ag-PTFE conductor showed a decreased sheet resistance due to the higher Ag volume fraction and better current path in the $\mathrm{Ag}$ network. However, as expected from Fig. 2d, the sheet resistance of the Ag-PTFE conductor prepared at a DC/ $\mathrm{RF}$ power ratio of $3 / 40 \mathrm{~W}$ was not measured because $\mathrm{Ag}$ failed to form the network, and there was no current path. The severely agglomerated $\mathrm{Ag}$ atoms, like isolated islands, are disconnected and cannot provide a current path. However, the Ag-PTEF hybrid conductor prepared at a higher DC power $(5 / 40,7 / 40 \mathrm{~W})$ with an increased $\mathrm{Ag}$ volume fraction showed sheet resistances of 3.09 and $17.23 \Omega$ /square, which indicated an effective Ag network current path in the PTFT matrix. Therefore, to fabricate high-performance stretchable Ag-PTFE conductors, 
achieving control over the DC/RF power ratio is a very important parameter in forming the Ag network in the PTFE matrix. We investigated the conduction mechanism of the Ag-PTFE conductor by measuring the change in resistance with a decrease in temperature (Fig. S3, Supplementary Information). The temperature coefficient of the resistivity measured from the Ag-PTFE conductor was found to be positive from 3 to $300 \mathrm{~K}$, indicating that the Ag-PTFE conductor possessed typical metallic characteristics due to the presence of the Ag network.

The microstructure of the Ag-PTFE conductor was analyzed using transmission electron microscopy (TEM). Bright-field (BF) TEM images of the Ag-PTFE hybrid conductor (DC/RF power ratio of 5/40 W) are shown in Fig. 3a, b for cross-sectional analysis. As shown in the SEM image of Fig. 2d, Ag atoms agglomerate in the PTFE matrix. In the BF TEM image of Fig. 3a, the selfassembled Ag atoms show different contrast due to junction or overlapping regions, as indicated by the arrow. Self-agglomerated Ag networks are well interconnected and form a conduction path embedded in an amorphous PTFE matrix. The enlarged cross-sections of the Ag-PTFE electrodes of Fig. 3b, c show that crystalline $\mathrm{Ag}$ is embedded in the amorphous PTFE matrix. Figure $3 \mathrm{~b}$

Table 1 Surface energy of the Ag-PTFE films with increasing sputter power.

\begin{tabular}{|c|c|c|c|c|}
\hline \multicolumn{2}{|l|}{ Power [W] } & \multirow{2}{*}{$\begin{array}{l}\text { Diiodomethane } \\
{\left[\mathrm{mJ} / \mathrm{m}^{2}\right] \text { (dispersive) }}\end{array}$} & \multirow{2}{*}{$\begin{array}{l}\text { DI-Water } \\
{\left[\mathrm{mJ}^{2} \mathrm{~m}^{2}\right]} \\
\text { (polar) }\end{array}$} & \multirow{2}{*}{$\begin{array}{l}\text { Surface } \\
\text { energy } \\
{\left[\mathrm{mJ} / \mathrm{m}^{2}\right]}\end{array}$} \\
\hline RF (PTFE) & $\mathrm{DC}(\mathrm{Ag})$ & & & \\
\hline \multirow[t]{3}{*}{10} & 3 & 24.35 & 43.35 & 67.70 \\
\hline & 5 & 22.85 & 42.92 & 65.77 \\
\hline & 7 & 17.31 & 43.01 & 60.32 \\
\hline \multirow[t]{3}{*}{20} & 3 & 17.28 & 42.95 & 60.23 \\
\hline & 5 & 12.84 & 43.60 & 56.44 \\
\hline & 7 & 12.54 & 42.79 & 55.33 \\
\hline \multirow[t]{3}{*}{40} & 3 & 9.98 & 39.60 & 49.58 \\
\hline & 5 & 9.57 & 38.98 & 48.55 \\
\hline & 7 & 6.26 & 38.73 & 44.99 \\
\hline
\end{tabular}

shows magnified high-resolution TEM (HRTEM) images of the Ag network on the PU substrate. The agglomerated Ag showed a crystalline structure, which was confirmed by the fast Fourier transform (FFT) pattern at a corner. Figure $3 \mathrm{c}$ also shows a magnified PTFE portion in the hybrid electrode. This showed that an amorphous PTFE matrix with a disarranged lattice existed around the crystalline Ag network.

Figure 4a shows the optical transmittance of the AgPTFE conductor in the visible wavelength region as a function of the DC/RF power ratio. Despite the existence of oblique Ag, the Ag-PTFE conductor showed semitransparent optical properties in the visible wavelength region due to the self-assembled network structure of the sputtered Ag. It was clearly shown that the optical transmittance of the Ag-PTFE electrode is mainly affected by the DC power applied to the Ag target because the PTFE matrix has very high optical transmittance, as shown in Fig. 4a. All Ag-PTFE films are semitransparent with optical transmittances of $25.27-38.49 \%$ at a transmittance wavelength of $550 \mathrm{~nm}$. Figure $4 \mathrm{~b}$ compares the optical transmittance of the Ag-PTFE electrodes according to the DC/RF power ratio. Due to the similar optical transmittance, it is difficult to distinguish the samples with the naked eye. Through the semitransparent AgPTFE conductors, we can observe the SKKU logo. Figure $4 \mathrm{c}$ is a photograph of the optimized Ag-PTFE conductor grown at a DC/RF ratio of $5 / 40 \mathrm{~W}$ in natural light. We also carried out continuous cycling stretching from 0 to $40 \%$ and measured the transmittance change, as shown in Fig. S4 and Table S1 (Supplementary Information). Even after $40 \%$ stretching, the Ag-PTFE electrode showed a constant optical transmittance change $(\Delta T)$, indicating good optical stability of the Ag-PTFE electrodes.

In general, a PU is a well-known elastomer with very weak intermolecular bonds, high viscosity, and low elastic coefficients. Due to these characteristics, transparent PU is an excellent stretchable substrate material and has been widely employed in wearable and stretchable devices ${ }^{30,31}$. We also used commercial transparent PU as a stretchable substrate instead of typical PDMS fabricated through a complicated transfer process. The mechanical properties of the Ag-PTFE film sputtered on the stretchable PU substrate were investigated, as shown in Fig. 5. The

Table 2 Sheet resistance and conductivity of the Ag-PTFE hybrid films with increasing DC/RF power.

\begin{tabular}{|c|c|c|c|c|c|c|c|c|c|}
\hline \multirow{2}{*}{$\begin{array}{l}\text { RF power (PTFE) } \\
\text { DC power (Ag) }\end{array}$} & \multicolumn{3}{|l|}{$10 \mathrm{~W}$} & \multicolumn{3}{|l|}{$20 W$} & \multicolumn{3}{|c|}{$40 W$} \\
\hline & $3 w$ & $5 \mathrm{~W}$ & $7 w$ & $3 w$ & $5 \mathrm{~W}$ & $7 \mathrm{w}$ & $3 w$ & $5 \mathrm{~W}$ & $7 w$ \\
\hline Sheet resistance $[\Omega /$ square $]$ & 4.83 & 3.43 & 3.09 & 6.94 & 4.64 & 3.24 & - & 17.23 & 13.30 \\
\hline Conductivity $[\mathrm{S} / \mathrm{cm}]$ & 103,519 & 145,772 & 161,812 & 72,046 & 107,758 & 154,321 & - & 29,019 & 37,593 \\
\hline
\end{tabular}

The 20-nm-thick pure Ag film sputtered at a DC power of $5 \mathrm{~W}$ shows a sheet resistance of $2.53 \Omega /$ square and a conductivity of $197,000 \mathrm{~S} / \mathrm{cm}$. 

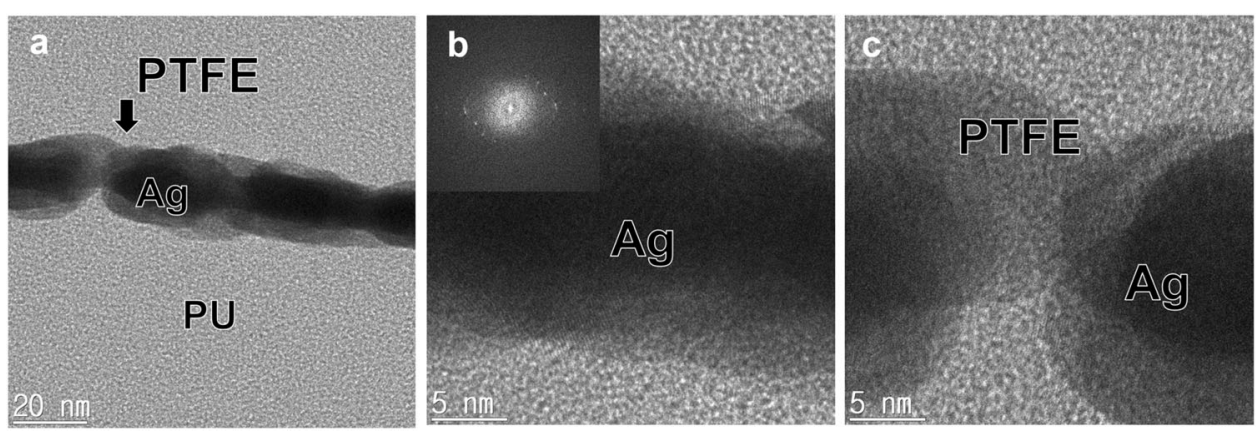

Fig. 3 Microstructure of the Ag-PTFE hybrid conductor on the PU substrate. a Cross-sectional TEM image of the Ag-PTFE hybrid conductor. b Magnified TEM image of the crystalline Ag network overlapping on the amorphous PTFE matrix with a fast Fourier transform (FFT) pattern of the Ag network. c Enlarged cross-sectional image of the Ag network embedded-PTFE matrix.

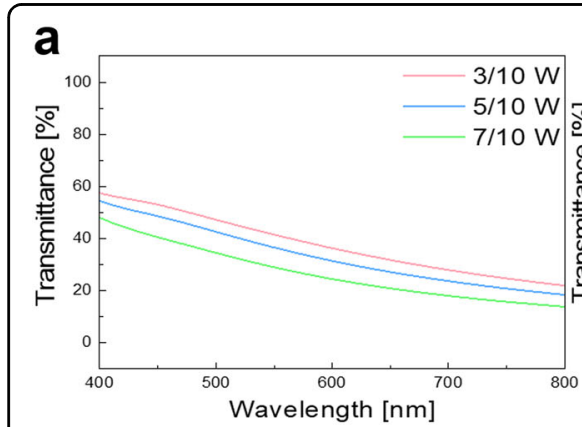

\section{b Ag/PTFE power ratio}
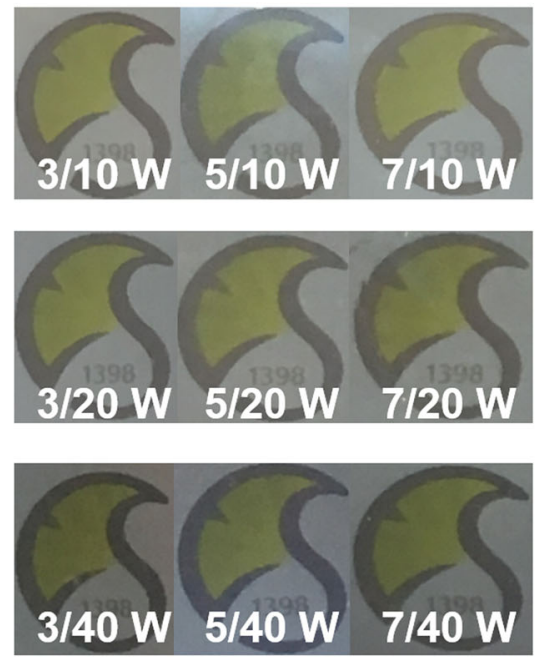
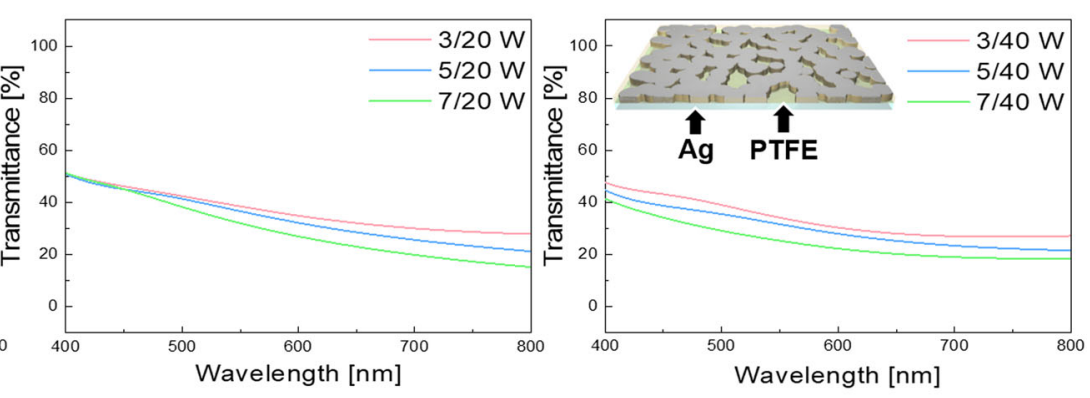

\section{C}

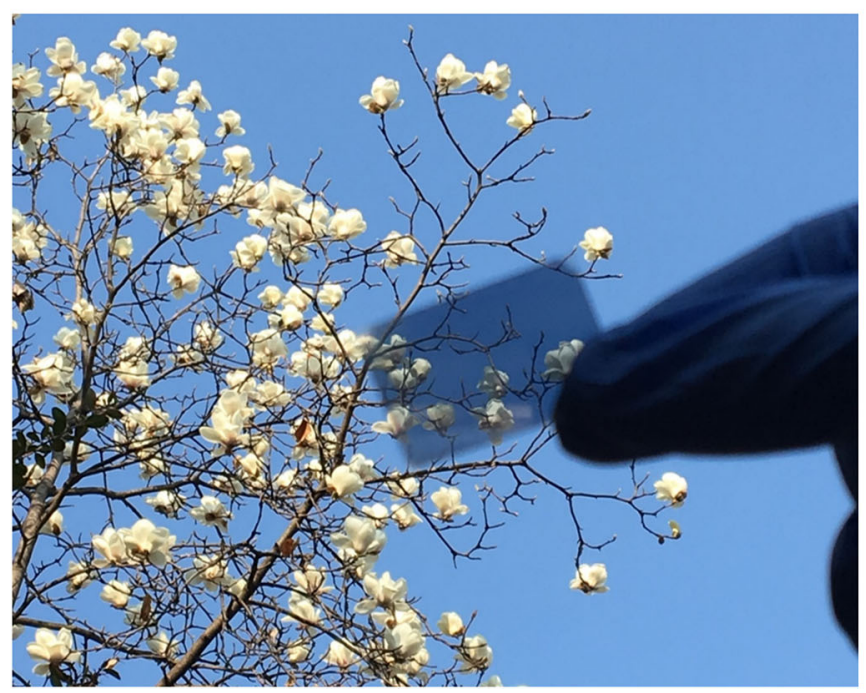

Fig. 4 Optical properties of the Ag-PTFE hybrid conductor on a PU substrate. a Optical transmittance of the Ag-PTFE conductor in the visible wavelength region with increasing sputtered DC (Ag target) and RF (PTFE target) power. b The images show the color and transparency of the AgPTFE hybrid conductor under each sputtered condition. c Photographs showing the semitransparent Ag-PTFE hybrid conductor on a PU substrate in natural light.

change in the resistance of the Ag-PTFE electrode on the PU can be expressed as $\Delta R=R-R_{0}$, where $R$ is the resistance measured in situ under stretching and $R_{0}$ is the resistance measured initially. In the stretching test, one clamp holding a $2 \times 3 \mathrm{~cm}^{2}$ sized sample was fixed, and the other clamp was moved laterally to produce a strain from 


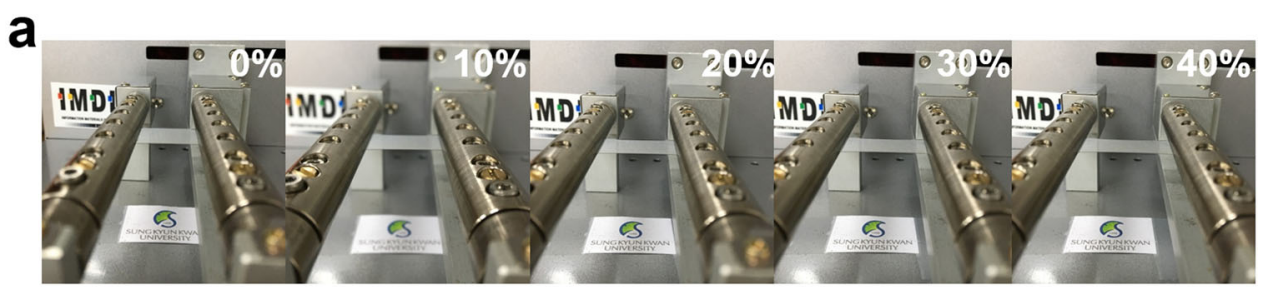

b

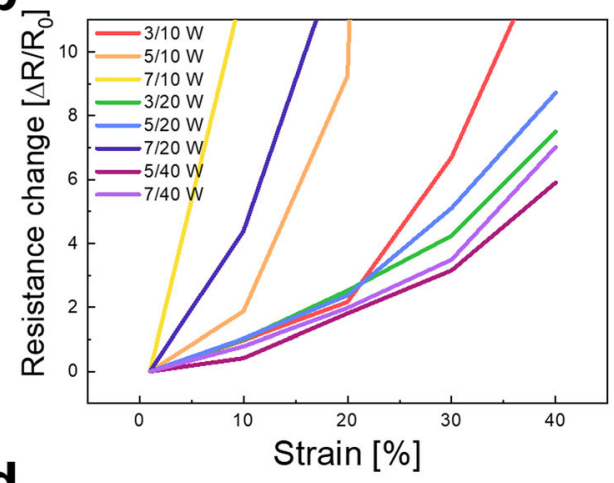

d

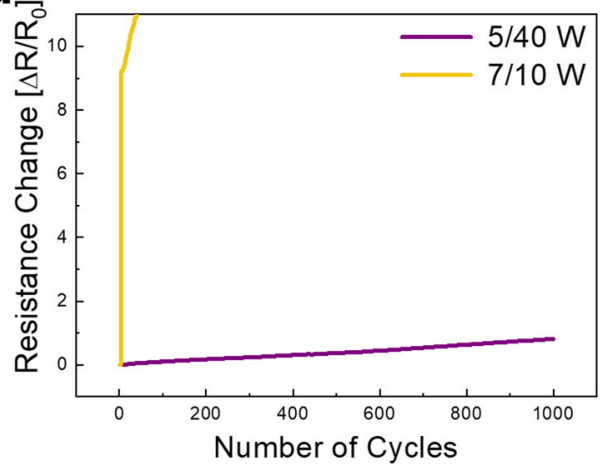

C

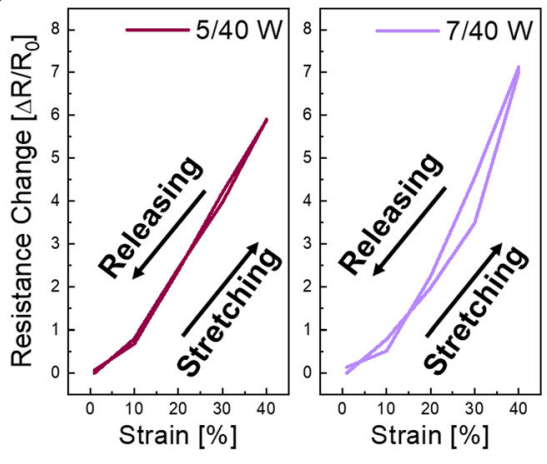

e
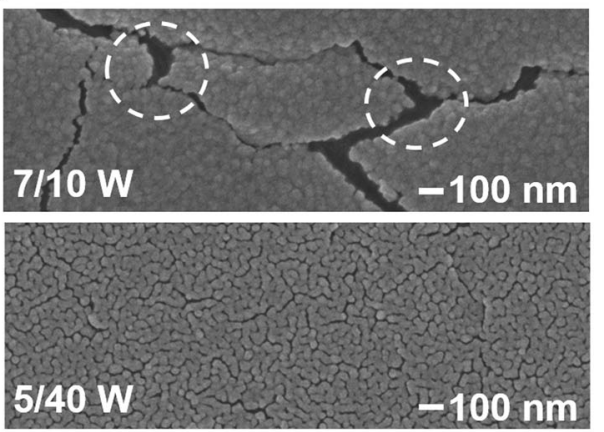

Fig. 5 Stretchability of the sputtered Ag-PTFE hybrid conductor. a The pictures show the stretching steps of the Ag-PTFE film up to a strain of $40 \%$ in a lab-made stretching system. $\mathbf{b}$ Change in resistance of the Ag-PTFE hybrid conductor as a function of the strain prepared at different DC/RF power ratios. c Hysteresis of the Ag-PTFE hybrid conductor prepared at different DC/RF ratios (5/40 W and 7/40 W). d Dynamic fatigue test of the AgPTFE hybrid conductor with the worst and best stretchability at a strain of 20\%. e Surface FESEM images of the Ag-PTFE hybrid conductor after 1000 cycles of repeated stretching in (d).

0 to $40 \%$ (Fig. $5 \mathrm{a}$ ). Figure $5 \mathrm{~b}$ shows the measured change in resistance of all sputtered Ag-PTFE conductors except the insulating sample grown at a DC/RF power ratio of $3 /$ $40 \mathrm{~W}$ with increasing strain. Among the Ag-PTFE samples, hybrid conductors with a large Ag volume fraction $(5 / 10 \mathrm{~W}, 7 / 10 \mathrm{~W}$, and $7 / 20 \mathrm{~W})$ showed a significant increase in resistance within a strain of $10 \%$, indicating severe crack formation or delamination of the Ag-rich conductors. However, the Ag-PTFE conductor with a large volume fraction of PTFE prepared at DC/RF power ratios of $5 / 40 \mathrm{~W}$ and $7 / 40 \mathrm{~W}$ showed a minimal change in resistance even at a strain of $20 \%$. The amorphous PTFE matrix with good stretchability could uniformly dispatch the external force when the PU substrate was stretched. Figure $5 \mathrm{c}$ compares the hysteresis of the change in resistance of the Ag-PTFE films prepared at DC/RF power ratios of $5 / 40 \mathrm{~W}$ and $7 / 40 \mathrm{~W}$ during stretching and releasing. Within a strain of $40 \%$, both samples showed similar stretchability and hysteresis during the increase and decrease in strain. A similar change in resistance in the hysteresis confirmed that the Ag-PTFE conductor with the high PTFE volume fraction could be applied to wearable devices. Figure $5 \mathrm{~d}$ shows a dynamic fatigue test for the best and worst samples prepared at DC/RF power ratios of $7 / 10 \mathrm{~W}$ and $5 / 40 \mathrm{~W}$ with increasing numbers of $20 \%$ stretching cycles. The Ag-PTFE conductor prepared at a DC/RF ratio of $5 / 40 \mathrm{~W}$ showed a small change in resistance, even after 1000 stretching cycles. However, the sample prepared at a DC/RF power of $7 / 10 \mathrm{~W}$ showed a rapid change in resistance within 4 cycles during a repeated $20 \%$ stretching test. Figure $5 \mathrm{e}$ shows a surface FESEM image of the Ag-PTFE samples prepared at DC/RF power 

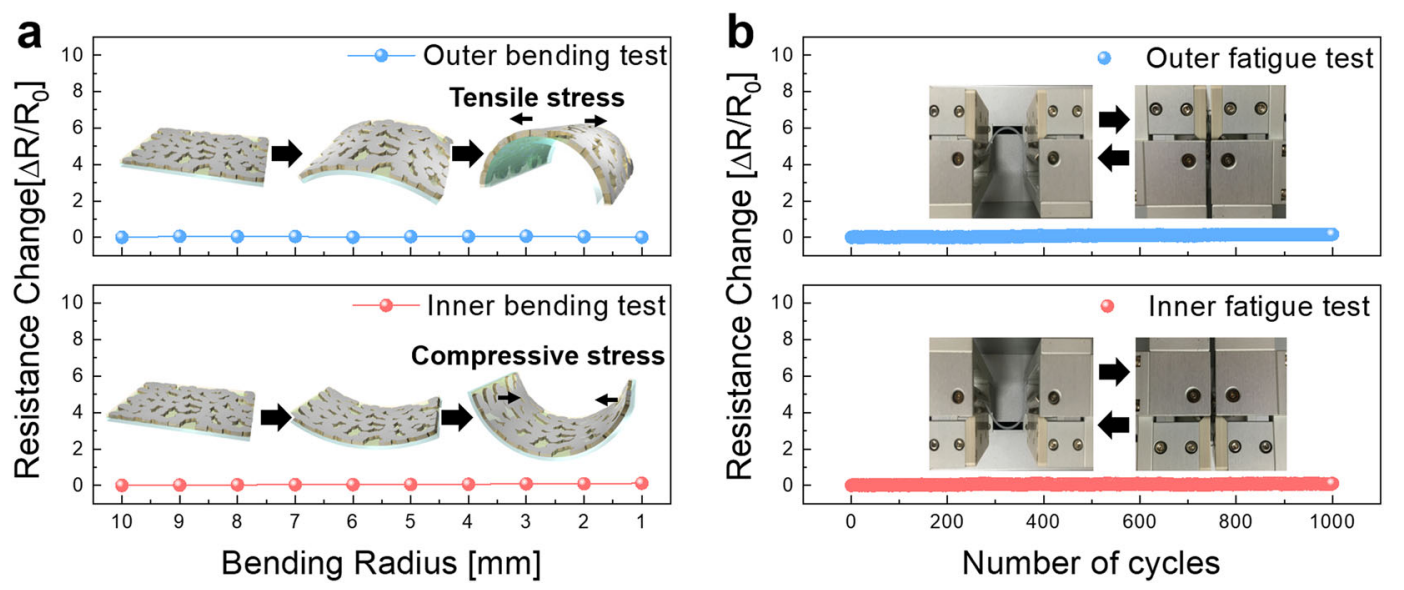

Fig. 6 Mechanical properties of the Ag-PTFE hybrid conductor. a The outward and inward bending test results for the Ag-PTFE hybrid conductor with decreasing outward (upper panel) and inward (bottom panel) bending radii. The inset demonstrates the outward and inward bending of the Ag-PTFE hybrid conductors. b Dynamic outward/inward bending fatigue test of the Ag-PTFE hybrid conductor at a fixed bending radius of $1 \mathrm{~mm}$ for 1000 repeat cycles.

ratios of $7 / 10 \mathrm{~W}$ and $5 / 40 \mathrm{~W}$ after 1000 cycles of stretching. As expected from Fig. 5d, the Ag-PTFE conductor prepared at a $\mathrm{DC} / \mathrm{RF}$ power ratio of $7 / 10 \mathrm{~W}$ showed severe cracks, as indicated by the dashed circles. Due to a high Ag volume fraction, the Ag-PTFE sample showed the easy formation of a crack, which is closely related to the increased resistance. Since the crack resulted in a physically separated Ag-PTFE conductor, however, the resistance of the conductor abruptly increased, even at $20 \%$ stretching. However, the Ag-PTFE sample prepared at a DC/RF power ratio of $5 / 40 \mathrm{~W}$ maintained the Ag network embedded in the PTFE matrix without cracks on the surface after 1000 cycles of $20 \%$ stretching. Figure 6a shows the results of the outer and inner bending tests of the optimized sample prepared at a $\mathrm{DC} / \mathrm{RF}$ power of $5 / 40 \mathrm{~W}$ with a decreasing bending radius. The outer and inner bending tests for the other samples are shown in Fig. S5 (Supplementary Information). As illustrated in the inset, the bending of the sample was performed by a horizontal motion of the clamp holding the samples. The horizontal movement of the clamp led to a decrease in the bending radius, with in situ measurements of the resistance of the electrode. The sample was bent outward when the Ag-PTFE film was clamped facing upward and bent inward when the sample was facing down. The outer bending sample experienced tensile stress, while the inner bending sample film experienced compressive stress. Regardless of the inner and outer bending modes, the Ag-PTFE conductor exhibited a constant resistance change as the bending radius decreased from 10 to $1 \mathrm{~mm}$ (Fig. 6a). This means that the Ag conduction pathway in the PTFE matrix is well connected under tensile/compressive stress by inward/outward bending. These results indicated that the
Ag-PTFE film can be easily applied in stretchable and wearable devices. Both the dynamic outer and inner bending tests in Fig. $6 \mathrm{~b}$ showed constant resistance during 1000 repeated cycles. The small bending radius and outstanding flexibility of the Ag-PTFE electrode demonstrate its potential for application in wearable and stretchable devices.

To demonstrate the potential of the sputtered Ag-PTFE conductors, we fabricated and tested a semitransparent, wearable and stretchable strain sensor, an interconnector, a touch screen sensor, and an EMG sensor. We showed the practicability of using the Ag-PTFE conductor for stretchable and wearable strain sensors. The stretchable and wearable strain sensor requires relatively high sensitivity and is one of the simplest and most efficient forms for reading applied strain based on the change in electrical resistance of the conductor ${ }^{32}$. In addition to the high stretchability and sensitivity, the long-term performance stability of these stretchable sensors is also critical for practical applications. The response in the resistance of the stretchable strain sensor fabricated using the AgPTFE conductor is shown in Fig. 7a, b. As shown in Fig. $7 \mathrm{a}$, the Ag-PTFE-based strain sensor showed a stable sensing performance under repeated stretching/release for 100 cycles at strains of $10 \%$ and $20 \%$. Generally, the GF of the strain sensor is the ratio of the relative change in resistance to the strain. Therefore, GF represents the sensitivity of the strain sensor as a measure of the change in resistance according to the strain ${ }^{33}$.

$$
\mathrm{GF}=\frac{\Delta R / R_{0}}{\varepsilon}=\frac{R-R_{0}}{R_{0} \times \varepsilon}
$$

where $\varepsilon$ is the strain applied to the sensor, $R_{0}$ is the initial resistance, and $R$ is the final resistance at a strain of $\varepsilon$. 


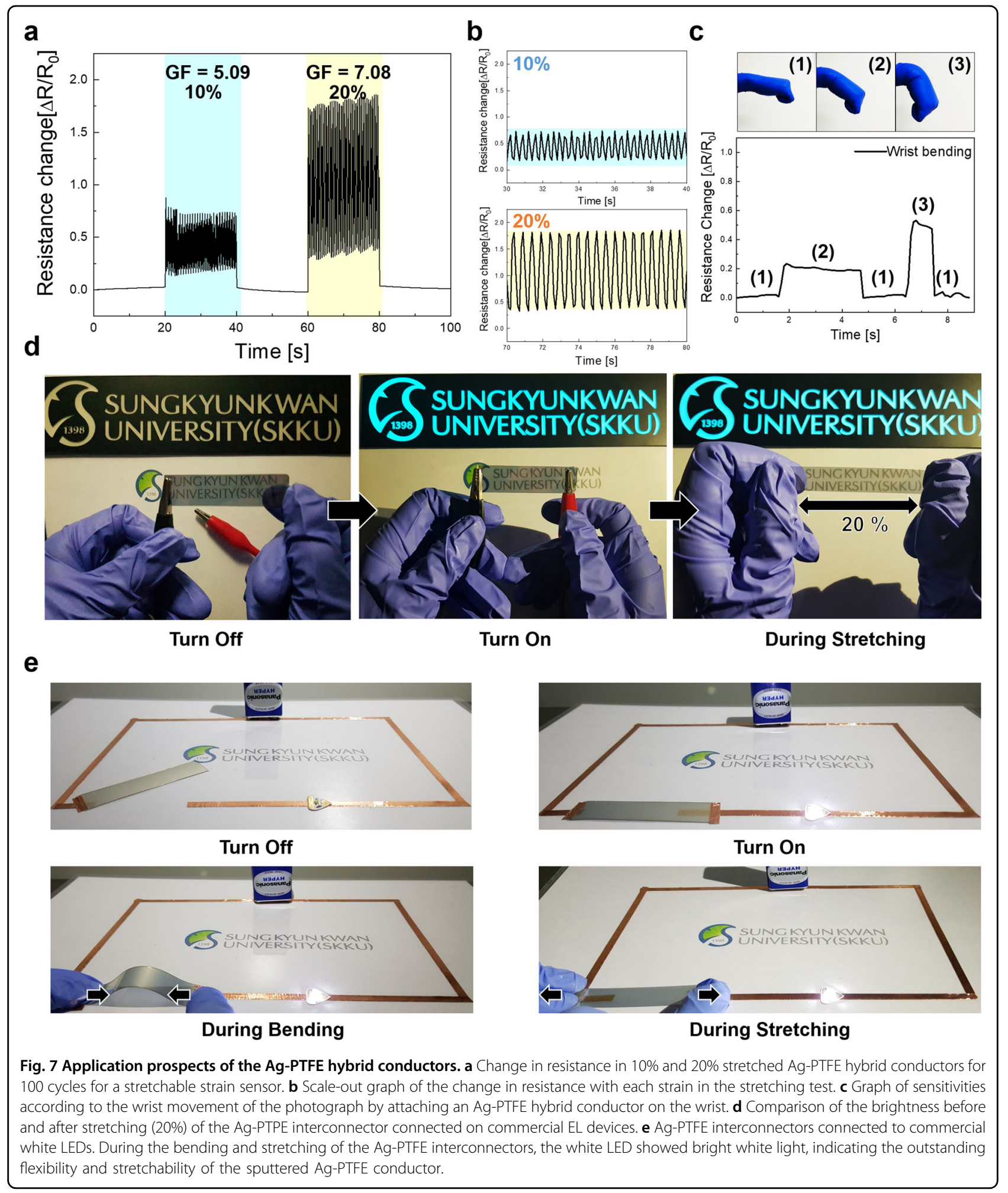

Figure $7 \mathrm{~b}$ shows the curve of the relative change in resistance $\Delta R / R_{0}$ versus time under 10 and $20 \%$ strain during 100 cycles of stretching tests. The GF values of the Ag-PTFE conductor-based strain sensors are 5.09 and
7.08 for $10 \%$ and $20 \%$ strain, respectively. As a result, the Ag-PTFE conductor-based strain sensor responds to strain with high sensitivity and acceptable linearity. With their high stretchability and sensitivity, Ag-PTFE strain 
sensors can be used successfully to monitor a wide range of human motion. A stretchable strain sensor attached to the wrist could accurately track repeated movements during bending, as shown in Fig. 7c. The resistance of the stretchable and wearable strain sensors increase sharply during bending of the wrist since bending at a larger angle generally causes greater strain. The strain sensor is sensitive enough to distinguish the degree of wrist bending, as shown in the upper panels. The sputtered Ag-PTFE conductor could be applied in stretchable interconnectors. The pictures in Fig. $7 \mathrm{~d}$ show the AgPTFE conductor applied as an interconnector for commercial EL devices. Two sides of the blue EL device with the SKKU logo were attached by the Ag-PTFE conductor. AC power was applied to turn on the EL device through the stretchable Ag-PFTE conductor. The Ag-PTFE hybrid electrode shows continuous lighting even at 20\% stretching, as shown in Fig. 7 d. The blue EL device is continuously lit even after $20 \%$ stretching due to the outstanding stretchability of the Ag-PTFE conductor. This indicated that the hybrid electrode has high conductivity even after 20\% stretching. Because the resistance of the interconnector connected to the EL is proportional to its length and inversely proportional to its cross-sectional area, the blue EL showed slightly decreased light intensity above $20 \%$ stretching. The AgPTFE conductor was also applied as a flexible and stretchable interconnector for the white LEDs, as shown in Fig. 7e. The LED connected with the Ag-PTFE interconnectors remained lit even after bending (bending radius of $5 \mathrm{~mm}$ ) and stretching of $20 \%$. The continuous lighting of the white LEDs connected to the stretched AgPTFE interconnector demonstrates promise as a stretchable interconnect for stretchable and wearable sensors.

To demonstrate the applicability of the developed AgPTFE conductor, we fabricated a capacitive-type stretchable touch sensor to measure the touch of a human, which was used as electronic skin and in a human monitoring system (Fig. S6) ${ }^{34,35}$. To use attachable touch panels on the human body, wearable touch sensors require good touch responsivity and high stretchability, similar to wearable strain sensors. Figure 8a shows the schematic structure of the touch sensor and multilayer for the touch panel. We used an Ag-PTFE conductor sputtered on the PU substrate as the bottom and top electrodes. The PU substrate was also used as a dielectric layer between the top and bottom Ag-PTFE conductors. Our touch sensor was used as a capacitive type where the signal comes from a change in the capacitance between the electrodes, as illustrated in Fig. 8b. As shown in Fig. $8 \mathrm{c}$, since contact with the touch panels led to a change in the capacitance, the sensor was able to sense human touch. The measurements of the capacitance responses $\left(\Delta C / C_{0}=\left(C-C_{0}\right) / C_{0}\right.$, where $C_{0}$ is the initial capacitance and $C$ is the change in the capacitance) for pristine and stretched states are shown in Fig. 8b. This touch sensor also exhibits stable sensing performance during stretching $(\varepsilon=20 \%)$ due to the outstanding stretchability of the Ag-PTFE conductor. The difference in capacitance change between the pristine state and the stretching state is caused by a decrease in the thickness of the dielectric layer of the touch sensor due to stretching. Therefore, it is possible to detect strain through the difference in capacitance change ( $C=\varepsilon A / d$, where $\varepsilon$ is the permittivity of the dielectric, $A$ is the area of the touch sensor, and $d$ is the distance between the electrode in the touch sensor). To confirm that there was no change in the capacitance due to electrode fatigue, we compared the base capacitance and confirmed that there was no change in electrode performance (Fig. S7, Supplementary Information). The capacitance responsivity values $\left(\Delta C / C_{0}\right)$ to touch stimuli applied on each sensor of the $3 \times 3$ arrays are illustrated in Fig. 8d. The results of the evaluation for each sensor in the array are shown in Fig. 8e. Most of the sensors in the array showed responsivity values close to $-23.2 \%$. We verified the suitability for use in stretchable and wearable touch sensors that can be worn, with touch detection even in the stretched state.

Furthermore, the Ag-PTFE conductor was applied to fabricate a stretchable EMG sensor. Figure 9a shows the fabrication process of the stretchable EMG sensor using the Ag-PTFE conductor. To enable stretchability in the sensor, nature-inspired strain-free mechanical structures were taken into account, as was reported recently ${ }^{36}$. This architecture with excellent sensing performance can be implemented with wearable smart sensors (Fig. 9b). Unlike conventional photolithography techniques that are time consuming, expensive, hazardous, and mask dependent, the laser provides the desired patterns inexpensively within fractions of a minute in a userfriendly manner with roll-to-roll processability ${ }^{37,38}$. Furthermore, patch-based sensors have drawn great attention to wearable electronics due to their breathable and long-term wearable forms ${ }^{39,40}$. In addition, the EMG sensor was confirmed to have been manufactured and used in various sensors. These structures provide mechanical flexibility and stretchability for the sensor, which can thereby. sustain a significant amount of deformation when subjected to complex stress environments. Figure 9b, c consists of a real image showing the position of the data obtained during the measurement of the proposed EMG sensor. Three sensors were placed on the forearm with the two ( + and - ) electrodes attached on top of the flexor muscle, which generally exhibits good potential during hand clenching. Then, the EMG signals were measured by a bipolar method using a Biopac system. Another electrode was placed far from the other two sensors and used as a ground electrode. To 

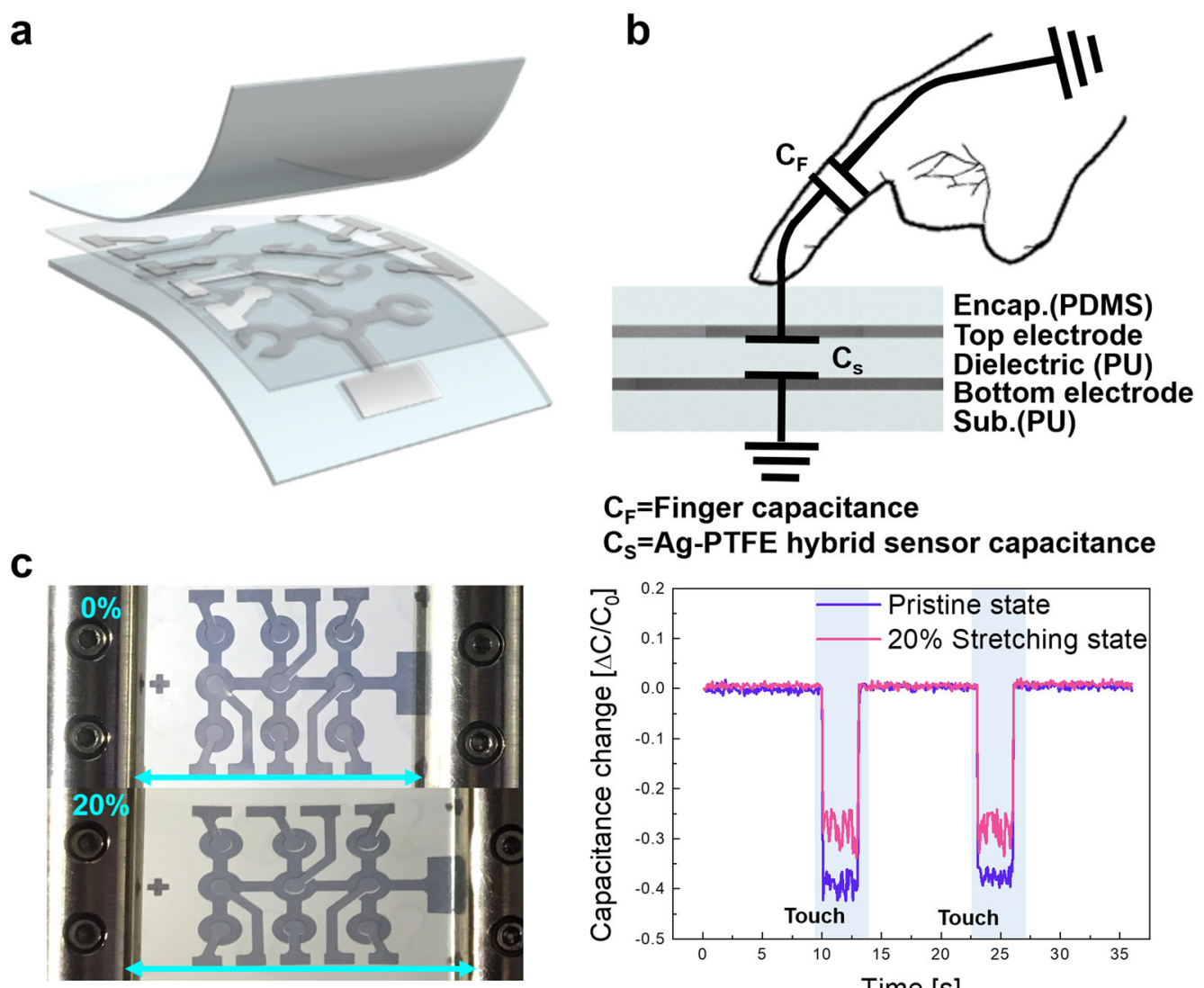

\section{$\mathrm{C}_{\mathrm{F}}=$ Finger capacitance \\ $\mathrm{C}_{\mathrm{S}}=\mathrm{Ag}-\mathrm{PTFE}$ hybrid sensor capacitance}
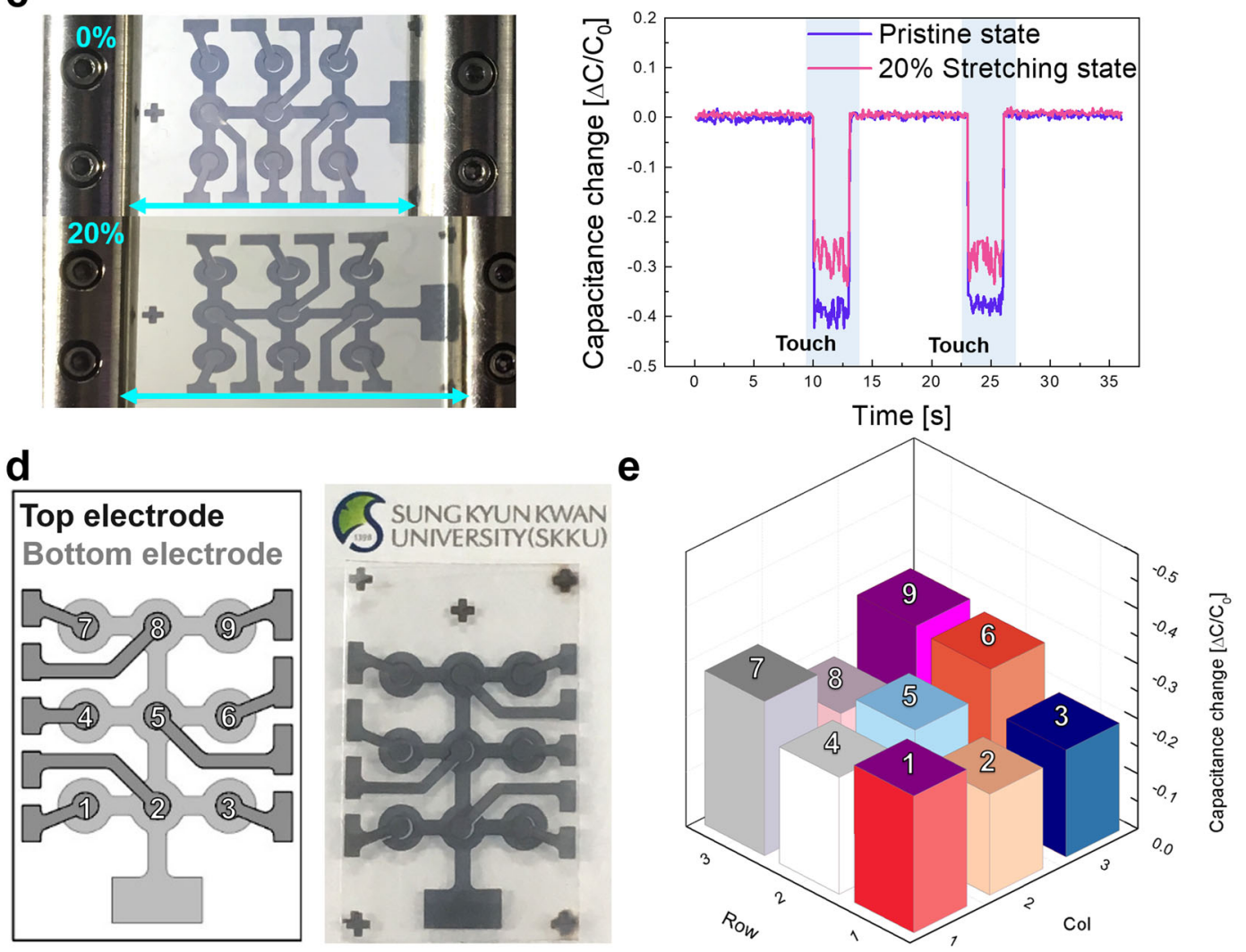

Fig. 8 Performance of stretchable touch sensor with Ag-PTFE hybrid conductors. a Schematics of a stretchable touch sensor using a patterned Ag-PTFE hybrid conductor. $\mathbf{b}$ Schematic illustration of a touch measurement with the five layers of the stretchable touch sensor labeled. $\mathbf{c}$ The pictures show the pristine and 20\% stretched touch panel. The change in capacitance indicates a touch stimulation in the pristine state and $20 \%$ stretching state. $\mathbf{d}$ Patterns and photographs of touch sensors, $3 \times 5 \mathrm{~cm}^{2}$. e Graph of the capacitance change between each touch array due to touch stimulation.

acquire the desired signals, a bandpass filter was applied (low pass filter $<500 \mathrm{~Hz}$, high pass filter $>35 \mathrm{~Hz}$ ) with a data sampling rate of $2 \mathrm{kHz}$. Overall, the sensor showed amplitudes with an average of $0.6 \mathrm{mV}$ with clear features when the forearm muscles were squeezed, with values that are generally comparable to those seen in previous reports (Fig. 9d).

\section{Conclusion}

In summary, we have demonstrated semitransparent, stretchable, and wearable Ag-PTFE hybrid conductors for stretchable and wearable interconnectors and sensors. Stretchable and wearable Ag-PTFE hybrid conductors were fabricated by a simple and mature cosputtering process with variations in the $\mathrm{DC} / \mathrm{RF}$ 


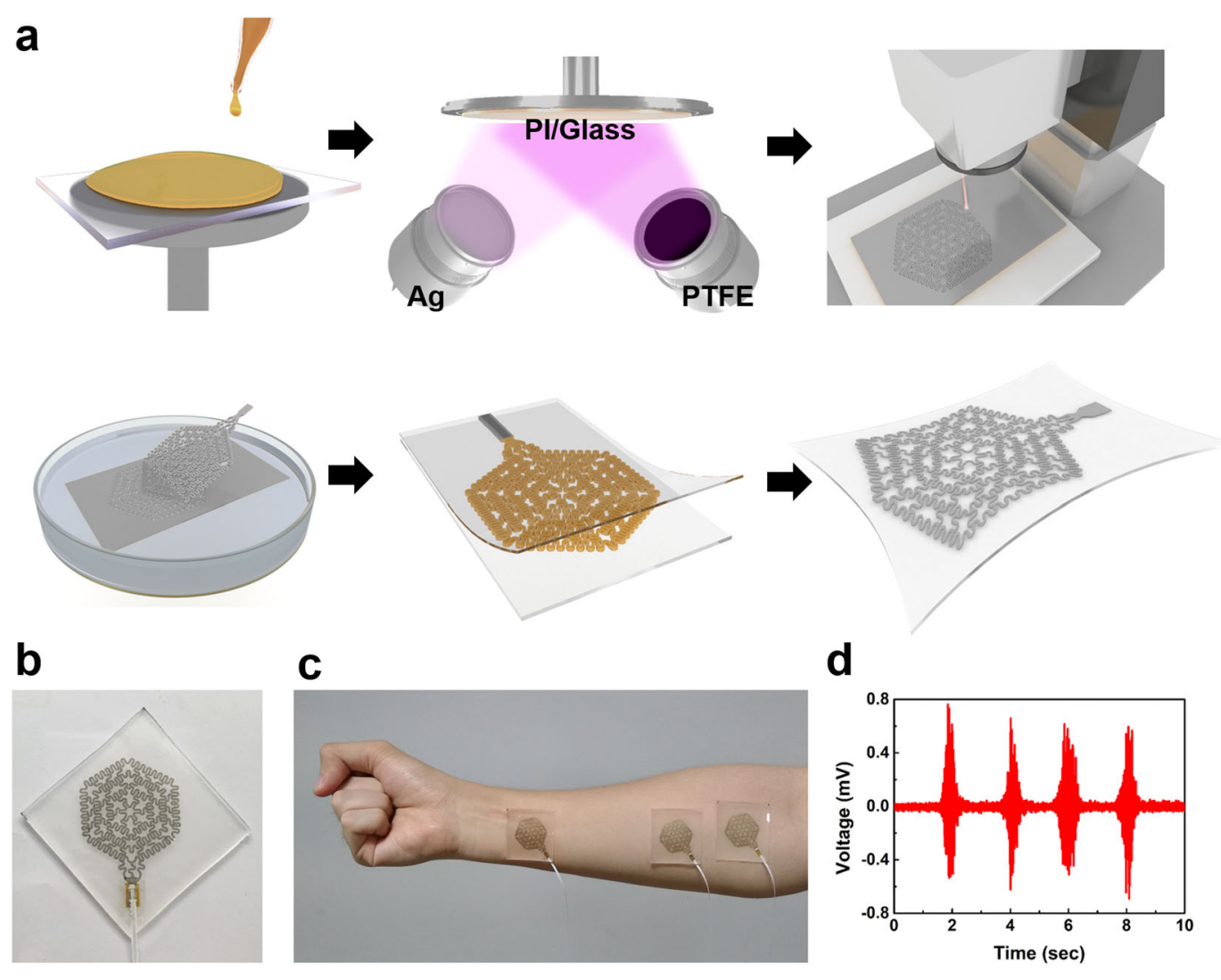

Fig. 9 Schematic illustration of the EMG sensor fabrication process. a Spin coating solution-processed Pl, deposition of Ag-PTFE conductor by sputtering, laser-processing of the Ag-PTFE/PI film into desired patterns, the immersion of the sensor in hot water $\left(90^{\circ} \mathrm{C}\right)$, ACF bonding followed by attachment of PDMS-PEIE substrate on top of the sensor design, and EMG sensor on stretchable PDMS-PEIE substrate. $\mathbf{b}$ Photograph of the EMG sensor. c Photograph of EMG sensors attached to the human body. $\mathbf{d}$ The positions and the obtained data during the measurement of the EMG sensor.

power ratio applied to the Ag and PTFE targets. The PTFE-modified Ag hybrid electrodes have a high stretchability of $0-40 \%$, a low sheet resistance of $3.09-17.23 \Omega$ /square, and a semitransmittance of 25.27-38.49\%, which are suitable values for wearable, stretchable, and self-cleaning sensors. In addition, it was confirmed that the Ag-PTFE conductor forms an incomplete wetting surface according to the content of PTFE, which is suitable for self-cleaning devices. The current path formed by the Ag network embedded in the insulator PTFE matrix can be attributed to the low sheet resistance and high mechanical stretchability. In addition, the PTFE matrix improved the mechanical properties of the Ag-PTFE electrode at relatively high strains. Furthermore, we showed the elasticity of the Ag-PTFE hybrid conductor to extreme mechanical strain, including stretching and inward/outward bending, to demonstrate the practicability of using Ag-PTFE hybrid conductors for stretchable electronics. Finally, we demonstrated the feasibility of using the Ag-PTFE hybrid conductor with a stretchable strain sensor, stretchable EL/LED interconnectors, stretchable touch sensor, and stretchable EMG sensor.

\section{Acknowledgements}

This work was supported by the Technology Innovation Program (or Industrial Strategic Technology Development Program) (10079601) funded by the Ministry of Trade, Industry \& Energy (MOTIE, Korea). This work is supported by Korea Electric Power Corporation (KEPCO, CX72170049).

\section{Author contributions}

Y.S. and H.-K.K. designed the experiments and prepared the manuscript. Y.J.K. Y.L., N.-E.L., Y.C.W., S.G., and S.K. performed the device evaluation. All authors discussed the results and commented on the manuscript.

\section{Conflict of interest}

The authors declare that they have no conflict of interest.

\section{Publisher's note}

Springer Nature remains neutral with regard to jurisdictional claims in published maps and institutional affiliations.

Supplementary information is available for this paper at https://doi.org/ 10.1038/s41427-020-00277-6 
Received: 3 June 2020 Revised: 28 October 2020 Accepted: 4 November 2020.

Published online: 8 January 2021

\section{References}

1. Wang, C. et al. Advanced carbon for flexible and wearable electronics. Adv. Mater. 31, 1801072 (2019).

2. Hong, $\mathrm{H}$. et al. Anisotropic thermal conductive composite by the guided assembly of boron nitride nanosheets for flexible and stretchable electronics. Adv. Funct. Mater. 29, 1902575 (2019).

3. Wei, P. et al. Flexible and stretchable electronic skin with high durability and shock resistance via embedded 3D printing technology for human activity monitoring and personal healthcare. Adv. Mater. Technol. 4, 1900315 (2019).

4. Park, S. J. \& Park, C. H. Suit-type wearable robot powered by shape-memoryalloy-based fabric muscle. Sci. Rep. 9, 9157 (2019).

5. Kim, Y. et al. Organic electrochemical transistor-based channel dimensionindependent single-strand wearable sweat sensors. NPG Asia Mater. 10, 1086-1095 (2018).

6. Zhang, C. et al. A stretchable dual-mode sensor array for multifunctional robotic electronic skin. Nano Energy 62, 164-170 (2019).

7. Herrmann, J. et al. Nanoparticle films as sensitive strain gauges. Appl. Phys. Lett. 91, 183105 (2007).

8. Pandey, M. et al. Dependence of ITO-coated flexible substrates in the performance and bending durability of perovskite solar cells. Adv. Eng. Mater. 21, 1900288 (2019).

9. Zhong, Z. W., Tang, R. W. L., Chen, S. H. \& Shan, X. C. A study of screen printing of stretchable circuits on polyurethane substrates. Microsyst. Technol. 25 339-350 (2019).

10. Lin, Y. et al. A highly stretchable and sensitive strain sensor based on graphene - elastomer composites with a novel double-interconnected network. J. Mater. Chem. C 4, 6345-6352 (2016).

11. Kim, Y.-S. et al. All-in-one, wireless, stretchable hybrid electronics for smart, connected, and ambulatory physiological monitoring. Adv. Sci. 6, 1900939 (2019).

12. Hwang, B.-U. et al. A transparent stretchable sensor for distinguishable detection of touch and pressure by capacitive and piezoresistive signal transduction. NPG Asia Mater. 11, 23 (2019).

13. Yang, C., Huang, Y., Cheng, H., Jiang, L. \& Qu, L. Rollable, stretchable, and reconfigurable graphene hygroelectric generators. Adv. Mater. 31, 1805705 (2019).

14. Zhang, R., Ding, J., Liu, C. \& Yang, E.-H. Highly stretchable supercapacitors enabled by interwoven CNTs partially embedded in PDMS. ACS Appl. Energy Mater. 1, 2048-2055 (2018)

15. Park, S., Lee, H., Kim, Y.-J. \& Lee, P. S. Fully laser-patterned stretchable microsupercapacitors integrated with soft electronic circuit components. NPG Asia Mater. 10, 959-969 (2018).

16. Tian, G. et al. Copolymer-enabled stretchable conductive polymer fibers. Polym. (Guildf.). 177, 189-195 (2019).

17. Albrecht, A. et al. Over-stretching tolerant conductors on rubber films by inkjet-printing silver nanoparticles for wearables. Polymer 10, 1413 (2018).

18. Yadav, K., Jha, S., Jassal, M. \& Agrawal, A. K. Internally coated highly conductive and stretchable AgNW-PU hollow fibers. Polymer 169, 46-51 (2019).

19. Catenacci, M. J., Reyes, C., Cruz, M. A. \& Wiley, B. J. Stretchable conductive composites from Cu-Ag nanowire felt. ACS Nano 12, 3689-3698 (2018).

20. Zhou, Y., Azumi, R. \& Shimada, S. A highly durable, stretchable, transparent and conductive carbon nanotube-polymeric acid hybrid film. Nanoscale 11, 3804-3813 (2019).
21. Fan, X. et al. A Transfer-printed, stretchable, and reliable strain sensor using PEDOT: PSS/Ag NW hybrid films embedded into elastomers. Adv. Mater. Technol. 3, 180030 (2018).

22. Wang, Y., Zhang, L. \& Wang, D. Ultrastretchable hybrid electrodes of silver nanowires and multiwalled carbon nanotubes realized by capillary- forceinduced welding. Adv. Mater. Technol. 4, 1900721 (2019).

23. Zhou, L-y.., Fu, J-z., Gao, Q., Zhao, P. \& He, Y. All-printed flexible and stretchable electronics with pressing or freezing activatable liquid-metal - silicone inks. Adv. Funct. Mater. 30, 1906683 (2019).

24. Woo, J. Y., Kim, K. K. Lee, J., Kim, J. T. \& Han, C.-S. Highly conductive and stretchable Ag nanowire/carbon nanotube hybrid conductors. Nanotechnology 25, 285203 (2014).

25. Montazerian, H. et al. Graphene-coated spandex sensors embedded into silicone sheath for composites health monitoring and wearable applications. Small 15, 1804991 (2019).

26. Kim, S. H. et al. Fluorocarbon thin films fabricated using carbon nanotube/ polytetrafluoroethylene composite polymer targets via mid-frequency sputtering. Sci. Rep. 7, 1451 (2017).

27. Xi, Y., Kaper, H. J., Choi, C.-H. \& Sharma, P. K. Tribological properties of microporous polydimethylsiloxane (PDMS) surfaces under physiological conditions. J. Colloid Interface Sci. 561, 220-230 (2020).

28. Gao, Y. et al. Wettability of pear leaves from three regions characterized at different stages after flowering using the OWRK method. Pest Manag. Sci. 74, 1804-1809 (2018).

29. Kwok, D. Y., Li, D. \& Neumann, A. W. Fowkes' surface tension component approach revisited. Colloids Surf. A Physicochem. Eng. Asp. 89, 181-191 (1994).

30. Sun, W.J. et al. Highly conductive and stretchable carbon nanotube/thermoplastic polyurethane composite for wearable heater. Compos. Sci. Technol. 181, 107695 (2019)

31. Zhai, Y. et al. Flexible and wearable carbon black/thermoplastic polyurethane foam with a pinnate-veined aligned porous structure for multifunctional piezoresistive sensors. Chem. Eng. J. 382, 122985 (2020).

32. $\mathrm{Xu}, \mathrm{H}$. et al. Dual-mode wearable strain sensor based on graphene/colloida crystal films for simultaneously detection of subtle and large human motions. Adv. Mater. Technol. 5, 1901056 (2020).

33. Fuji, Y. et al. An ultrasensitive spintronic strain-gauge sensor and a spin-MEMS microphone. Electron Commun. Jpn. 102, 48-54 (2019).

34. Cataldi, P. et al. Carbon nanofiber versus graphene-based stretchable capacitive touch sensors for artificial electronic skin. Adv. Sci. 5, 1700587 (2018).

35. Shi, R., Lou, Z., Chen, S. \& Shen, G. Flexible and transparent capacitive pressure sensor with patterned microstructured composite rubber dielectric for wearable touch keyboard application. Sci. China Mater. 61, 1587-1595 (2018).

36. Chae, $\mathrm{H}$. et al. Laser-processed nature-inspired deformable structures for breathable and reusable electrophysiological sensors toward controllable home electronic appliances and psychophysiological stress monitoring. ACS Appl. Mater. Interfaces 11, 28387-28396 (2019).

37. Wang, Y., Hu, H., Shao, J. \& Ding, Y. Fabrication of well-defined mushroomshaped structures for biomimetic dry adhesive by conventional photolithography and molding. ACS Appl. Mater. Interfaces 6, 2213-2218 (2014).

38. Yoo, J.-H., Park, J. B., Ahn, S. \& Grigoropoulos, C. P. Laser-induced direct graphene patterning and simultaneous transferring method for graphene sensor platform. Small 9, 4269-4275 (2013).

39. Chen, T. et al. Triboelectric self-powered wearable flexible patch as 3D motion control interface for robotic manipulator. ACS Nano 12, 11561-11571 (2018).

40. Li, X. \& Sun, Y. A wearable button-like system for long-term multiple biopotential monitoring using non-contact electrodes. Smart Heal. 11, 2-15 (2019) 